\title{
Lineage-specific regulators couple cell lineage asymmetry to the transcription of the Caenorhabditis elegans POU gene unc-86 during neurogenesis
}

\author{
Ralf Baumeister, ${ }^{1,2}$ Yanxia Liu, ${ }^{1}$ and Gary Ruvkun ${ }^{1,3}$ \\ ${ }^{1}$ Department of Molecular Biology, Massachusetts General Hospital and Department of Genetics, Harvard Medical School, \\ Boston, Massachusetts 02114 USA
}

The POU homeo box gene unc-86 specifies neuroblast and neural identities in the developing Caenorhabditis elegans nervous system. After an asymmetric neuroblast division, unc-86 is expressed in one of two daughter cells in 27 lineage classes that are not obviously related by function or position. We show here that unc-86 transcriptional regulatory regions detect cell lineage asymmetry to activate unc-86 expression in one of two neuroblast daughter cells. Distinct regulatory regions activate unc-86 expression in particular sets of sublineages. Therefore the unc-86 regulatory region integrates distinct cell lineage asymmetry cues to activate unc-86 expression in the many classes of neuroblast cell lineages. In agreement with such lineage-specific regulation of unc-86 asymmetric activation, mutations in lin-11 (LIM homeo box), ham-1, and lin-17 affect the asymmetry of unc-86 expression in particular cell lineages, and mutations in lin-32 (achaete/scute family), $v a b-3$ (Pax-6 homolog) and $e g l-5$ ( $A b d-B$ homolog) affect the establishment of unc-86 expression in other cell lineages. Homologs of unc-86 and many of these unc-86 regulators have been implicated in control of neurogenesis in vertebrates and invertebrates. These data suggest that unc-86 acts in a phylogenetically conserved pathway that couples neuroblast cell lineage asymmetry to the generation of diverse neural types.

[Key Words: POU homeo domain protein; C. elegans; neurogenesis; unc-86; asymmetric cell division; transcriptional regulation]

Received September 27, 1995; revised version accepted April 15, 1996.

Transcription factors that are expressed in complex patterns have been shown to control key events in neural development (Jan and Jan 1994). These transcription factors regulate the pattern of neurogenesis in spatial domains (Doe et al. 1988; Skeath et al. 1992; Salser et al. 1993) or in stereotyped cell lineages (Finney et al. 1988; Miller et al. 1992; Jarman et al. 1993). They also regulate the detailed features of neural type such as patterns of synaptic connectivity (Miller et al. 1992) and the expression of signaling pathways in particular neural types (Desai et al. 1988; Johnson and Hirsh 1990; McIntire et al. 1993; Jin et al. 1994). They regulate the expression of many downstream effector genes (Xue et al. 1992) that actually mediate the complex recognition and signaling events during neurogenesis and neural function. The expression pattern of these transcription factor genes define where and when particular neural types are generated, or where and when specific events in neurogenesis occur. The control of their activation in complex pat-

\footnotetext{
${ }^{2}$ Present address: Laboratory of Molecular Biology/Genzentrum, Ludwig-Maximilians-University, D-81375 Munich, Germany.

${ }^{3}$ Corresponding author.
}

terns that presage key neurogenic events is a fundamental problem in neurogenesis.

unc-86 has such a central role in Caenorhabditis elegans neurogenesis. unc-86 couples asymmetry in neuroblast cell lineages to the generation of particular neuroblast and neural types during neural development (Finney and Ruvkun 1990). unc-86 mutations perturb neuronal cell lineages by disrupting the generation of mother/daughter asymmetry. In wild type, asymmetric neuroblast divisions generate two daughter cells that differ from one another and from their mother. In unc-86 mutants, one neuroblast daughter of particular asymmetric divisions retains the cell identity normally associated with its mother, whereas the other daughter cell is not affected. This leads to cell lineage reiterations that generate extra copies of some neurons and fail to generate other neurons. unc- 86 mutations also cause defects in other neurons that are not associated with cell lineage aberrations (Desai et al. 1988).

unc-86 encodes a POU-domain transcription factor (Finney et al. 1988; Ruvkun and Finney 1991). UNC-86 protein is expressed in 57 out of the 302 neurons of the C. elegans nervous system, including particular sensory 
neurons, motor neurons, and interneurons, some of which are known to be defective in unc-86 mutants (Fig. 1) (Finney and Ruvkun 1990). These neurons are not obviously related by function or position. However, the disparate cell lineages that express unc- 86 have one thing in common. unc-86 expression is initiated in only one of two daughter cells shortly after a cell division. Therefore, asymmetric expression of UNC- 86 protein must be sensitive to some factor segregated or activated asymmetrically at division, and unc- 86 must act in the daughters to couple this cell lineage asymmetry to neuroblast and neuronal cell fates.

We show here that the unc-86 transcriptional regulatory region detects cell lineage asymmetry, and that distinct regulatory regions mediate the asymmetric activation of unc-86 in particular domains of its expression pattern. We also identify genes that regulate the establishment of unc-86 asymmetric expression in particular cell lineages. Taken together, these data show that the unc-86 transcriptional regulatory region responds to multiple cell lineage asymmetry cues to build up the complex unc-86 expression pattern piece by piece. Based on spatial clustering and orientation of neuronal cell lineages that activate unc-86 expression, we present a model that local signals induce the asymmetric distribution or activation of unc- 86 regulators.

\section{Results \\ Asymmetric transcriptional regulation of unc- 86 expression in neural cell lineages}

To distinguish whether asymmetry of unc-86 expression is generated by transcriptional or translational control mechanisms, we constructed a series of fusion genes that bear various regions of the unc-86 gene and surveyed their expression in transgenic animals. Both $\operatorname{lac} Z$ and GFP fusions were used for this analysis. Expression patterns of at least three independent transgenic lines for each fusion gene were analyzed. Generally, these transgenes are expressed only in the 57 neurons or their precursor neuroblasts that normally express unc-86.

A 10,390-bp fragment from cosmid C30A5 that contains the entire unc-86 transcript plus $5.1 \mathrm{~kb}$ upstream of the unc-86 transcriptional start site and $1.8 \mathrm{~kb}$ downstream of the probable polyadenylation signal, rescues an unc-86(n846) null mutant for mechanosensation, dye uptake by IL2 neurons, and chemotaxis (see Fig. 3, be-
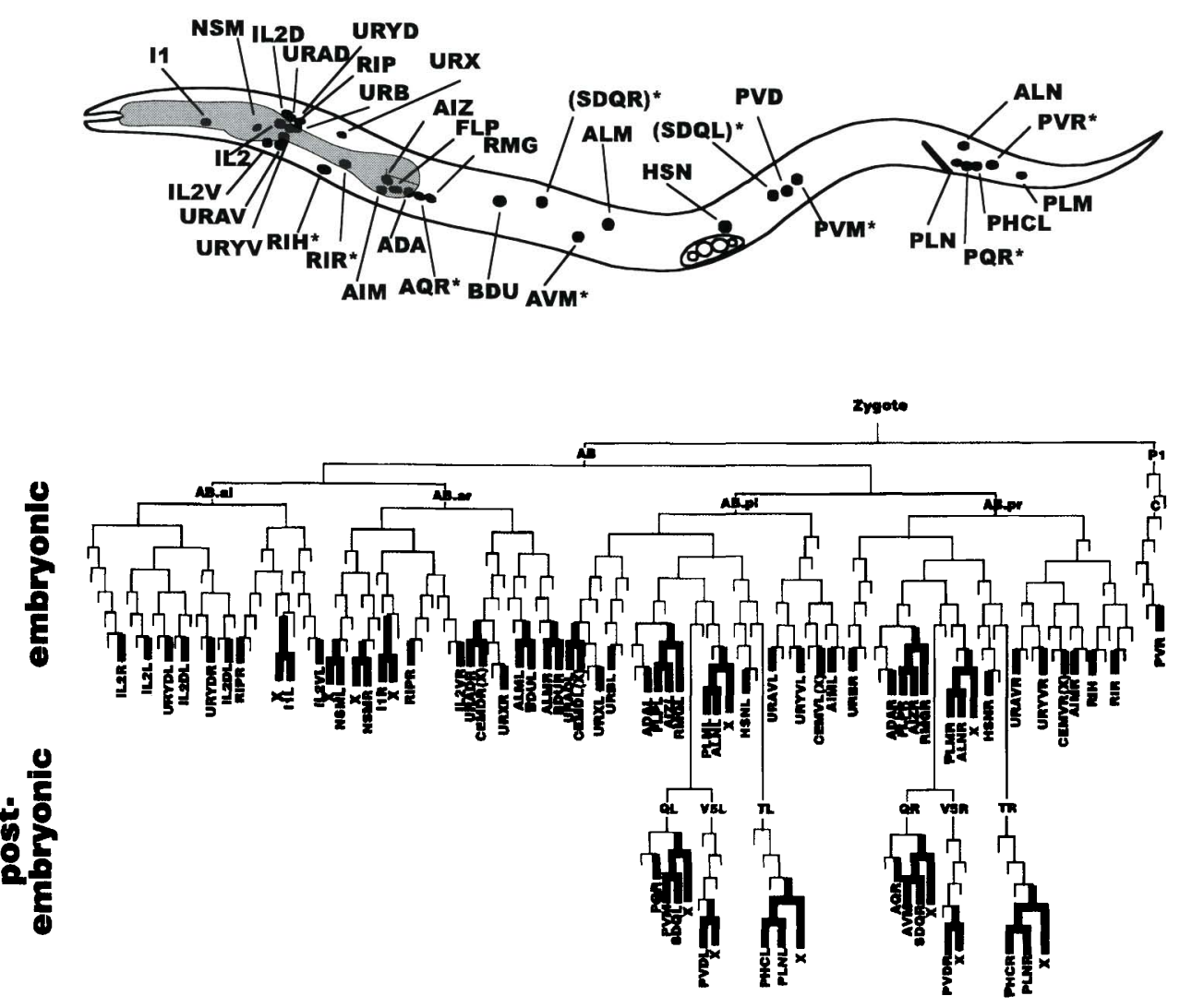

Figure 1. Position and lineage origin of cells expressing UNC-86 protein. (Top) Positions of neuronal nuclei that express unc-86 are shown (solid ellipses). For bilaterally symmetrical cells, only one is shown. ( $)$ Cells at asymmetric positions. Brackets at SDQ(L/R) indicate that unc-86 expression fades in late $\mathrm{Ll}$ as it is no longer visible afterwards. (Bottom) Lineage origin of unc-86 expressing cells. Neurons and neuroblasts that express unc- 86 in hermaphrodites are indicated by thick, solid lines. Modified from Finney and Ruvkun (1990). Lineage branches not leading to unc-86-expressing cells have been trimmed. (X) Programmed cell death. 
low) (Finney et al. 1988; R. Baumeister and G. Ruvkun, unpubl.). Rescue of egg-laying defects was not scored because of a similar defect associated with the rol-6 transformation marker. Transgenic unc-86(n846) animals bearing this unc-86 DNA fragment express Unc-86 protein in the normal set of 57 neurons, whereas no expression of UNC-86 is detectable in unc-86(n846) animals (Fig. 2).

Fusion genes bearing a segment from $5.1 \mathrm{~kb}$ upstream of the unc-86 transcription start to +52 of the unc- 86 mRNA (fusion gene $\mathrm{ABC}_{1234}$, see Fig. 3 ) are expressed in wild-type animals in the same 57 neurons that normally express unc-86 (Figs. 3A and 4A). Because unc-86 encodes a transcription factor that could regulate its own expression, this fusion gene expression could either reflect an autoregulatory response to endogenous chromosomal unc-86 gene activity, or a response to regulatory factors that normally initiate asymmetric unc-86 expression. To disable any autoregulation, we determined the pattern of fusion gene expression in an unc-86 null mutant that expresses no endogenous UNC-86 protein (Figs. 3 and 4 .

We found that unc- 86 does autoregulate and that only in absence of autoregulation could the activity of enhancers for the establishment of unc-86 expression be detected. The same fusion gene extrachromosomal arrays analyzed in wild type were transferred by mating to unc-86 null mutants. The determination of patterns of unc-86 reporter gene activation in an unc-86 mutant is complicated by the neurogenesis defects in this mutant. In those cell lineages where unc-86 is first expressed in a nondividing differentiating neuron, reporter gene expression in these neurons was recognized by the position of the cell bodies and processes (Fig. 3A). In those cell lineages where unc-86 is first expressed in neuroblasts, these neuroblasts generate reiterated sets of recognizable neurons in an unc-86 mutant that express particular fusion genes (Fig. 3A). The $-5.1-\mathrm{kb}$ to +52 -bp unc- $86 \mathrm{fu}-$ sion $\left(\mathrm{ABC}_{1234}, \mathrm{Fig} .3 \mathrm{~A}\right)$ is initially expressed in unc-86 mutant animals in the complete set of neuroblasts and neurons that initiate unc-86 expression in wild type (Fig. $4 \mathrm{C})$. Derivatives of this fusion gene that delete overlapping 41 and 45 nucleotide segments of the 52 nucleotides from the unc-86 mRNA $\left(\mathrm{ABC}_{1234 \Delta} 1 \mathrm{ABC}_{1234 \Delta 2}\right.$, Fig. 3B) are also expressed in all neurons that normally express unc-86. The expression levels in unc-86 mutant embryos are similar to those in wild-type embryos. However, both $\beta$-galactosidase and GFP expression levels from fusion genes decrease dramatically at post-L1 stages in unc-86 null mutants, in contrast to wild type.

Therefore, the $5.1 \mathrm{~kb}$ upstream of the unc- 86 transcript is sufficient to establish unc-86 expression in the correct neuroblasts and neurons but cannot maintain that expression in the absence of unc-86 gene activity. These data show that asymmetric expression of unc- 86 in these neural cell lineages is based on asymmetric regulation of unc-86 transcription rather than, for example, asymmetric segregation or activation of the unc- 86 mRNA, because no segments in the unc- 86 mRNA are essential to recapitulate normal unc- 86 regulation.

\section{Lineage-specific establishment of the asymmetric unc-86 expression pattern}

Dissection of the unc-86 transcriptional regulatory region revealed that distinct regulatory segments are acti-
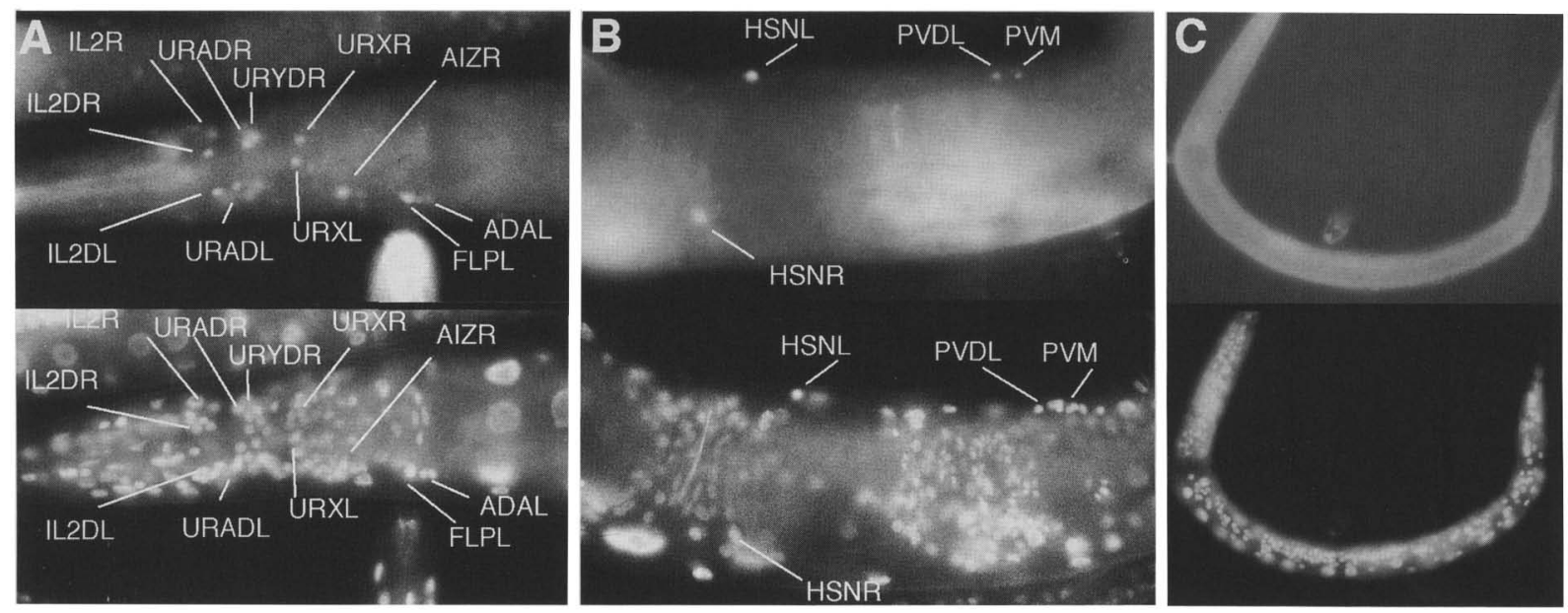

Figure 2. A 10,390-bp transgenic fragment containing the unc-86 gene rescues the unc-86(n846) null phenotype. (Top) Immunostaining with Unc-86-specific antibody. (Bottom) Nuclear DAPI staining. (A) unc-86(n846); Ex $\left(\mathrm{ABC}_{1234}\right.$ WXYZ) animal (for nomenclature, see legend of Fig. 3) containing the 10,390-bp unc-86 transgene expresses UNC-86 protein in a normal manner and is wild type for unc-86- mediated behaviors. Shown is normal expression of unc-86 in the anterior ganglion and deirid neurons. $(B)$ An adult unc$86(n 846) ; \operatorname{Ex}\left(\mathrm{ABC}_{1234} \mathrm{WXYZ}\right)$ animal showing normal HSN up-regulation of unc-86 expression. The expression of unc-86 in the HSN $(\mathrm{L} / \mathrm{R})$ is much brighter than that of the postdeirid neurons PVD and PVM; all these neurons express similar levels of Unc-86 at earlier stages (R. Baumeister and G. Ruvkun, unpubl.). (C) No expression of unc-86 can be detected in the unc-86(n846) null mutant animal. 
vated in specific cell lineages to assemble the complex unc-86 expression pattern. In an unc-86 null mutant, unc-86 fusion genes bearing distinct segments of the regulatory region (Fig. 3A) were activated in subsets of the unc-86 expression pattern. For example, the $\mathrm{BC}_{1234} \mathrm{fu}-$ sion gene (Fig. $3 \mathrm{~A}$ ) that bears the $-2.8-\mathrm{kb}$ to $+52-\mathrm{bp}$ unc-86 region is expressed in $\mathrm{L} 1$ stage animals in a subset of the normal unc-86 expression pattern (Fig. 4E). It is expressed in RIH, RIR, but not in the I1, NSM, HSN, or in the neuroblasts Q.p, V5.paap, and T.ppp. In contrast, the $\mathrm{AC}_{1234}$ fusion gene (Fig. 3A) that bears -5.1 - to $-2.7-\mathrm{kb}$ and $-0.8 \mathrm{~kb}$ to $+52 \mathrm{bp}$ is expressed in the neurons I1, NSM, HSN, and in the neuroblasts Q.p, V5.paap, and T.ppp, but is not expressed in RIH or RIR (Fig. 4F). Both fusion genes are expressed in other cell lineages (Fig. 3A).
These unc-86 regulatory segments are also sufficient to confer expression on reporter genes bearing an unrelated promoter. mec-7/GFP fusion genes bearing unc-86 DNA segments from the $-5.1-$ to $-2.8-\mathrm{kb}$ region (segment $A^{\prime}$ ) or the $-2.7-$ to $-0.8-\mathrm{kb}$ region (segment $\mathrm{B}^{\prime}$ ) cloned upstream of the probable mec-7 proximal promoter element were expressed in sets of neurons in an unc-86 mutant that are nearly identical to the analogous promoter deletion constructs bearing the corresponding unc-86 segments (Fig. 3A and $4 \mathrm{~J}-\mathrm{N}$ ). The $-2.7-$ to $-0.8-$ $\mathrm{kb}$ segment functioned in both orientations; only the normally oriented -5.1 - to -2.8 -kb segment was tested for regulatory activity (data not shown). The basic mec$7 /$ GFP reporter gene into which unc-86 DNA segments were placed is weakly expressed at late stages in two tail neurons and AVK in an unc-86(n846) mutant and in
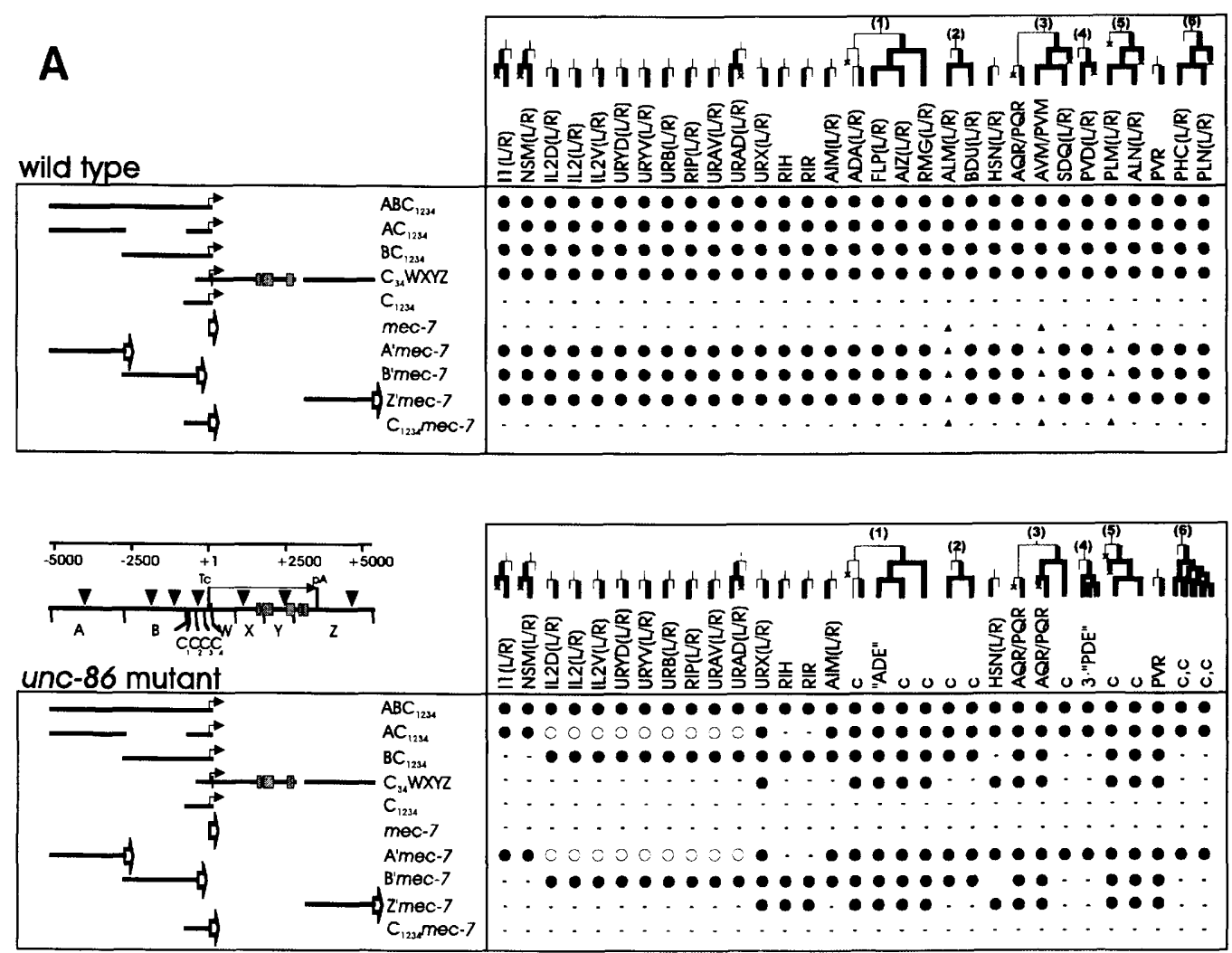

B
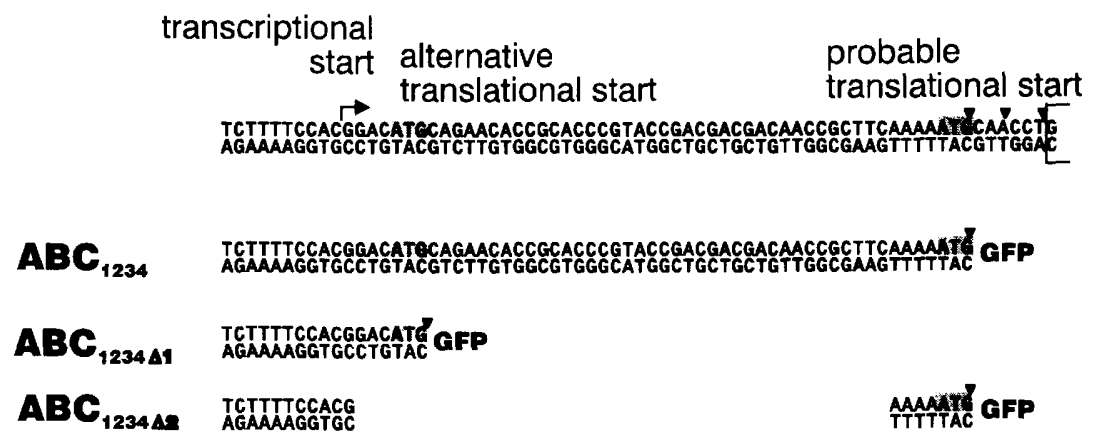

Figure 3. (See facing page for legend.) 
these neurons plus the six touch neurons in wild type (Fig. 3A). Therefore unc-86 DNA segments specifically confer GFP expression in other neurons.

These data show that the $-5.1-$ to -2.8 -kb unc- 86 region bears enhancers that are necessary and sufficient to activate unc-86 expression in the Il, NSM, HSN, Q.p, V5.paap and T.ppp neuroblasts and neurons, whereas enhancers in the $-2.6-$ to $-0.8 \mathrm{~kb}$ segment are necessary and sufficient to activate unc-86 expression in the RIR and RIH neurons. Enhancers for the activation of unc-86 expression in other cell lineages are located in both segments (Fig. 3A). No unc-86 regulatory activity was detected in the -754- to +52-bp DNA segment (Fig. 3A), that is likely to bear the proximal unc-86 promoter elements. This particular proximal region is not required for asymmetric unc-86 activation because unc-86 regulatory segments can activate expression of the $\mathrm{mec}-7 \mathrm{re}-$ porter gene bearing other proximal elements (Fig. 4).

The region $3^{\prime}$ to the unc-86 start site also contains segments that can activate asymmetric expression of unc-86 in particular neural cell lineages in unc-86 null mutants. Addition of the $+53-$ to $+5.2-\mathrm{kb}$ segment to the inactive -422 - to +52 -bp fusion gene (fusion gene $\mathrm{C}_{34} \mathrm{WXYZ}$, Fig. $3 \mathrm{~A}$ ) activates expression of this reporter gene in particular neural lineages but not in others. Expression was absent in the cell lineages that generate anterior sensory neurons but present in many cell lineages located more posteriorly (Figs. $3 \mathrm{~A}$ and $4 \mathrm{H}, \mathrm{I}$ ). Derivatives of this fusion gene missing various segments mapped the major enhancer activity for these cell lineages to the $+2.8-$ to $+5.3-\mathrm{kb}$ segment $(\mathrm{Z}$ in Fig. $3 \mathrm{~A})$. The $+2.8-$ to $+5.3-\mathrm{kb}$ unc-86 segment in either orienta- tion was also sufficient to confer expression in a similar set of neurons on the heterologous mec-7/GFP fusion gene in an unc-86 null mutant (Figs. $3 \mathrm{~A}$ and $4 \mathrm{~N}$ ).

None of the fusion genes bearing fragments of the unc86 regulatory domain express ectopically when transformed at low-copy number. However, high-copy transgenes revealed an example of negative regulation of asymmetric unc-86 expression in the $\mathrm{ADL}$ neurons that is not dependent on functional UNC-86 protein (Fig. 4G). unc-86 expression in ADL may be under negative regulation by a repressor that can be titrated.

\section{Multiple unc-86 autoregulatory regions}

Unlike the distinctive expression patterns in an unc-86 null mutant exhibited by fusion genes bearing different segments from the unc-86 promoter, the pattern of expression in unc-86 $+\mid$ animals bearing these fusion genes are identical. Each fusion gene is expressed in all 57 neurons that normally express unc- 86 and expression continues at all developmental stages (Figs. 3A, 4A,D,H,K). unc-86 DNA segments from these regions are also sufficient to confer to reporter genes bearing unrelated proximal elements expression in the 57 neurons that normally express unc-86: addition of the $-5.1-$ to $-2.8-\mathrm{kb}$ $\left(\mathrm{A}^{\prime}\right)$, the $-2.7-$ to $-0.8-\mathrm{kb}\left(\mathrm{B}^{\prime}\right)$, or the $+2.8-$ to $+5.3-\mathrm{kb}$ (Z) unc-86 segments to either mec-7/GFP or pes-10/lacZ fusion genes activates expression of these reporter genes in the entire unc-86 expression pattern (Figs. 3A and $4 \mathrm{~K}$ ). Therefore, there are multiple unc- 86 autoregulation sites distributed over regions that also bear cell-lineage-specific enhancers for the establishment of unc-86 expres-

Figure 3. unc-86 regulatory segments bear cell lineage-specific and autogenous regulatory activities. $|A|$ Promoter fragments used in the GFP and lacZ fusions are shown schematically to the left and their patterns of expression in wild-type (top right) and in an unc-86 null mutant (bottom right) are indicated. The 10.4-kb unc-86 DNA fragment that complements an unc-86/n846) null allele is shown at left, in the center. The genomic organization of unc-86 is depicted. Transcription (Tc) start and putative poly(A) site (pA) are indicated; (shaded boxes) exons. Segments used in the promoter analysis are labeled A (-5098:SpeI to - 2737:BglII), A' 1-5098:SpeI to -2809 :HindIII), B (-2737:BglII to $-635:$ BamHI), B' $\left(-2669\right.$ :HindIII to -754 :HindIII), $\mathrm{C}_{1}-\mathrm{C}_{4}(-754:$ HindIII to $+52 \mathrm{ATG})$, W (+127:XbaI to +836:HindIII), X (+836:HindIII to + 1784:StyI), Y (+1784:StyI to +2748:SpeI), Z (+2784:SpeI to + 5293:EcoRI). Segment labels were used to name the unc- 86 fusion genes. Also shown are the unc-86 segments fused to mec-7/GFP reporter genes, indicated by large arrowheads. Expression of unc-86 reporter genes at the left is indicated on the right by a solid circle under each of the sublineages from Fig. 1 that activate expression of unc-86. These sublineages are displayed from anterior to posterior. Thick lines indicate expression of UNC-86 in those sublineages. (O) Expression of the particular fusion gene in the particular sublineage. $(0 / \mathrm{Weak}$ expression. For wild type (top right panel), the sublineages are exactly as shown in Fig. 1. For unc-86 mutant animals (bottom right panel), lack of unc-86 gene activity alters many neuroblast cell lineages, as indicated. (1) AB.p(1/r)apaaa "deirid" lineage. (2) AB.arpp(a/ plapp "ALM/BDU" lineage. (3) Q(L/R) lineage. (4) V5(L/R).paa "postdeirid" lineage. (5) AB.p(1/r)apappp "PLM/ALN" lineage. (6) $\mathrm{T}(\mathrm{L} / \mathrm{R})$.pp lineage. (X) Programmed cell death. (c) Compact nucleus indicative of neural fate [lineage aberrations are described in Chalfie et al. (1981)]. The basic mec-7/GFP reporter gene without added unc-86 DNA segments is expressed in the mechanosensory neurons ALM, AVM/PVM, and PLM in wild-type $(\boldsymbol{\Delta})$, but because expression of mec-7 is dependent on unc-86, it is not expressed in these cell lineages in an unc-86 mutant. Therefore the regulatory activities of unc-86 DNA segments could not be scored in the three sublineages that generate mechanosensory neurons in wild type but could be scored in these altered lineages in an unc-86 mutant. Potential unc-86-binding sites indicated by inverted triangles in the center diagram (consensus sequence: CATnnnA/TAAT) (Xue et al. 1992; Li et al. 1993) are located at position -3950 (segment A), -1668 , and $-1214(\mathrm{~B}),-231(\mathrm{C}),+1085(\mathrm{X}),+2391(\mathrm{Y})$, and +4386 (Z). Segments A, B, and $Z$ in unc- 86 are each sufficient to respond to endogenous UNC-86 protein in all 57 neurons, suggesting that the potential binding sites on these segments may be functional. $(B)$ Deletion of sequences in the transcribed region do not affect the expression pattern of unc- 86 promoter-GFP reporter genes. The sequence flanking the unc-86 transcription start is shown. The mRNA start is indicated by an arrow, the two possible in-frame translational start codons are shown with a shaded background. Inverted triangles indicate the reading frame; the bracket at right indicates the splice site of the first exon. Deletion of these sequences results in weaker expression levels, which could reflect a less than optimal translational intitation signal caused by changes in the sequence flanking the ATG . 


\section{Baumeister et al.}

sion (Fig. 3A). The distinction between the expression pattern of these reporter genes in an unc-86 mutant vs. wild type suggest that these autoregulatory sites can respond to asymmetrically activated endogenous Unc-86 protein regardless of whether the fusion gene bears regulatory regions necessary for the initial activation of unc-86 expression in a particular cell lineage. For example, the fusion gene Z'mec-7/GFP (Fig. 3A) can only establish unc-86 expression in a small number of neurons but can respond to endogenous unc-86 activity in all 57 neurons that express the gene.

Regulators of asymmetric unc-86 expression

Because our analysis showed that asymmetric expres- sion of unc- 86 is transcriptionally based and that distinct segments in the unc-86 promoter regulate its expression in particular cells, we surveyed candidate mutants for perturbations in the expression of unc-86 in any or all of the sublineages that express the gene, as determined by immunofluorescent detection of UNC-86 protein (Finney and Ruvkun 1990). This analysis revealed genes that regulate the symmetry or activation of unc-86 expression in particular subdomains of its expression pattern.

lin-11 controls the asymmetry of unc-86 expression in the cell lineages that generate RIR

lin-11 controls cell lineage asymmetry in the ectoderm
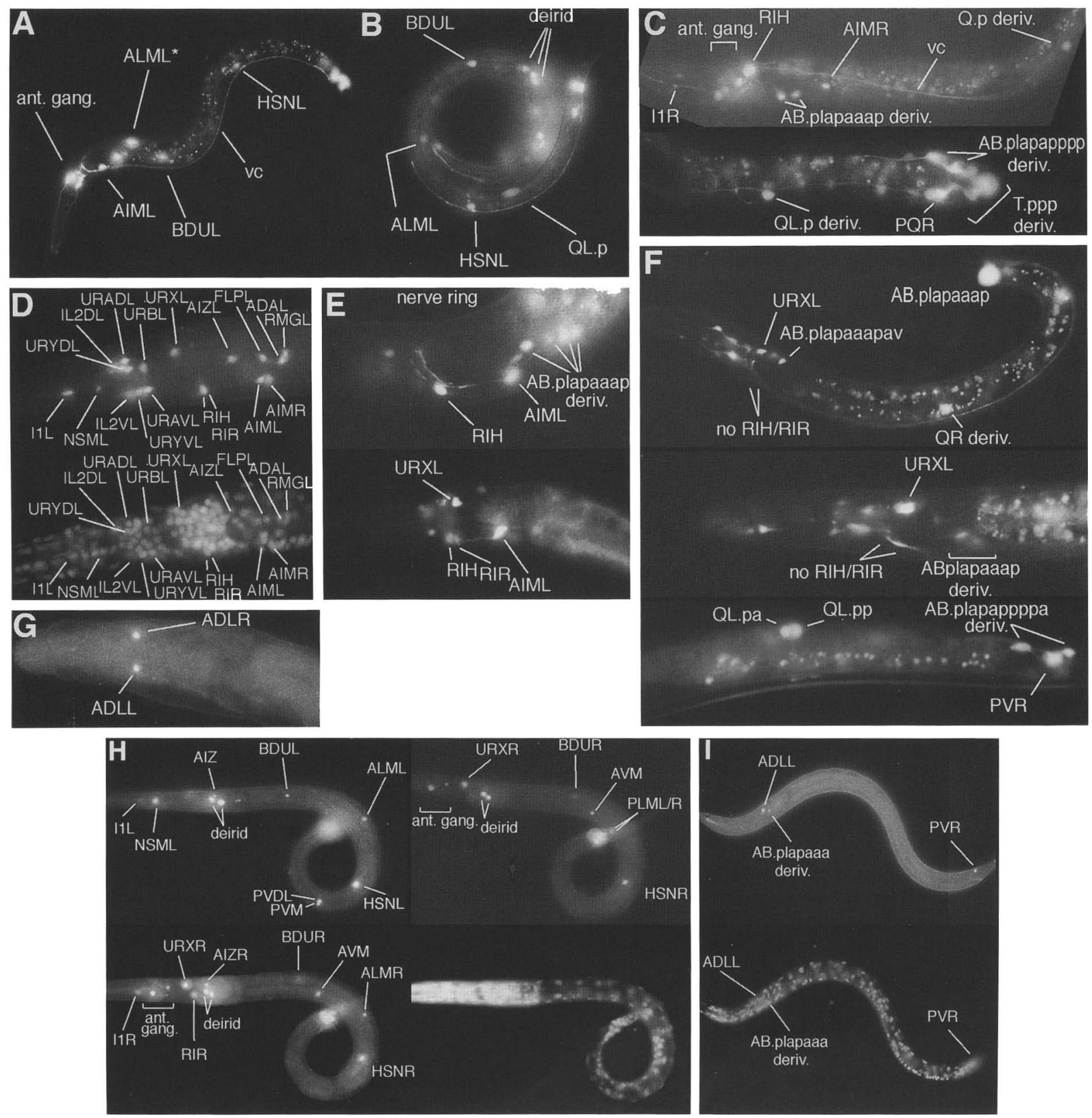

Figure 4. (See facing page for $\mathrm{J}-\mathrm{N}$ and legend.) 
(Freyd et al. 1990). We find ectopic expression of unc-86 in the interneuron AVG in animals carrying a strong lin-11(n389) allele (Figs. 5A and 6). unc-86 expression was detected in the AVG interneuron at all stages in more than $50 \%$ of the animals surveyed ( $n=90$ ). AVG is the sister of the unc-86-expressing interneuron RIR. Therefore, normal lin-11 activity prevents the symmetric expression of unc-86 in both AVG and RIR, presum-

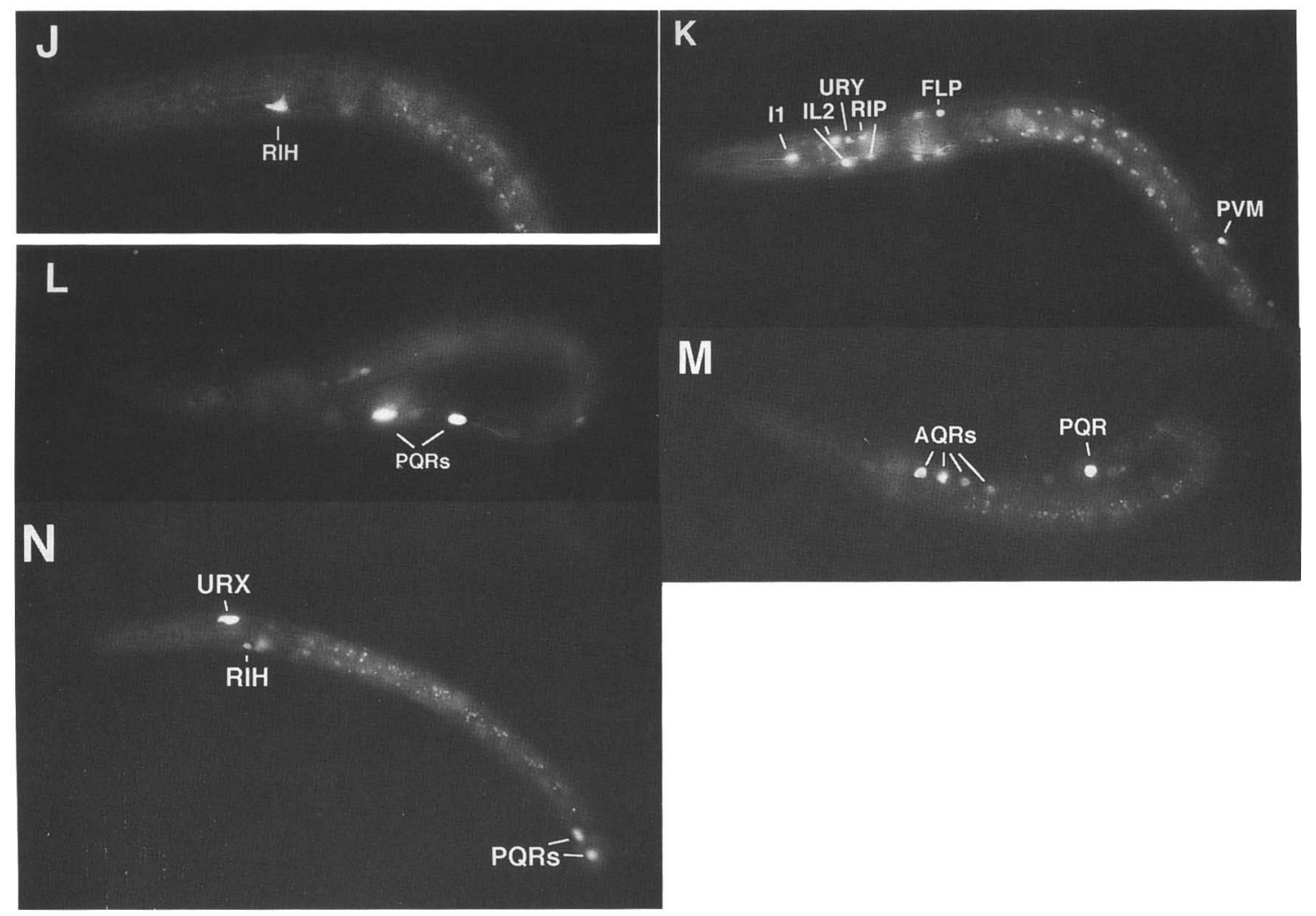

Figure 4. Expression of $l a c Z$ and GFP reporter constructs in wild-type and unc-86 mutant animals. $(A, B)$ Expression of a GFP fusion gene bearing the $-5.1-\mathrm{kb}$ to +52 -bp region of unc-86 (fusion gene $\mathrm{ABC}_{12.34} \mathrm{GFP}$ ) in wild-type $\mathrm{L} 1$ animals in all neurons. Both cell bodies and neural processes are fluorescent. The animal in $A$ shows expression of GFP from the unc-86 promoter in the anterior ganglion neurons, in a mismigrated $\left({ }^{\circ}\right)$ ALM neuron, the BDU, and HSN neurons. Shown in $B$ is expression of this fusion gene in the deirid neurons, BDU, ALM, HSN, and Q.p. (C) The same - 5.1-kb to + 52-bp GFP fusion gene $\left(\mathrm{ABC}_{1234} \mathrm{GFP}\right)$ in an unc-86(n846) mutant. (Top) Ventral view, showing expression of GFP in the sensory neurons of the anterior ganglion, Il, RIH, AIM, Q.p, and AB.pll/r)apaaap. Because of the rol-6(su1006) injection marker, the animal is twisted along its longitudinal axis. (vc) ventral cord. (Bottom) Expression of GFP in Q.p, T.ppp, and AB.plapapppp cell lineages that generate the tail neurons. (Left lateral view) Late $\mathrm{L} 1$ animal. (D) A - 5.1-kb to +52 -bp lac $Z$ fusion gene $\left(\mathrm{ABC}_{1234}\right.$ lac $Z$ fusion gene $)$ in a wild-type animal is expressed in all neurons that normally express unc-86. (Top) Anti- $\beta$-galactosidase antibody staining. (Bottom) DAPI. (E, F) Comparison of the -2.7 -kb to +52 -bp GFP fusion gene (BC 1234 ) and the $-5.1-\mathrm{kb}$ to $-2.7-\mathrm{bp},-0.8-\mathrm{kb}$ to $+52-\mathrm{bp}$ GFP fusion gene $\left(\mathrm{AC}_{1234}\right)$ expression patterns in unc-86/n846) animals. $(E) \mathrm{BC}_{1234}$ activates GFP expression in the ventral ganglion neurons $\mathrm{RIR}$ and $\mathrm{RIH}$ and in other neurons. $\langle F| \mathrm{AC}_{1234}$ is expressed in the Q, V5.paap, and T.ppp (tail) lineages and head neurons, but not in RIR and RIH. (G) Expression of a -0.4 - to +2.8 -kb lac $Z$ fusion gene $\left(\mathrm{C}_{34} \mathrm{WXY}-\mathrm{lacZ}\right.$ ) at high copy in adult unc-86(n846) animals, dorsal view. The only two neurons that express $I a c Z$ are ADL(L/R). No transgene expression was detected in transgenic wild-type animals bearing $\mathrm{C}_{34} \mathrm{WXY}$ at low-copy number or $\mathrm{C}_{1234}$ at low-or high-copy number. (H,I) Establishment and maintenance of unc- 86 expression function independently. Comparison of lacZ expression pattern of $\mathrm{C}_{34} \mathrm{WXYZ}$ in L2 wild-type with unc-86(n846) animals. $(H) \mathrm{C}_{34} \mathrm{WXYZ}$ is expressed in all 57 neurons in wild-type background. Three focal planes of the same animal and DAPI staining. $(I)(T o p)$ In the particular unc-86(n846) animal, $\mathrm{C}_{34}$ WXYZ is expressed in PVR, a neuron at the AIZ position [derived form AB.p(L/R/apaaa], and, ectopically in ADL head neurons. (Bottom) DAPI staining. (J) Expression of the $\mathrm{B}^{\prime} \mathrm{mec}-7 / \mathrm{GFP}$ fusion gene bearing the -2.7 to $-0.8 \mathrm{~kb}$ unc-86 segment in the RIH neuron in an unc-86(n846) animal. (K) The same -2.7 - to $-0.8-\mathrm{kb} \mathrm{B}$ 'mec-7/GFP reporter gene in wild type is expressed in all 57 neurons that express unc-86. Shown are the subset of these neurons that express in this particular animal. $(L)$ Expression of the A'mec-7/GFP fusion gene bearing the $-5.1-$ to -2.8 -kb unc-86 segment in the abnormal Q lineage in unc-86(n846) mutant animals is evinced by the expression of GFP in reiterated PQR neurons. $(M)$ The $\mathrm{B}^{\prime}$ mec-7/GFP fusion gene is also expressed in the reiterated $\mathrm{Q}$ lineages that generate the AQR and PQR neurons of an unc-86/n846) animal. (N) A Z'mec-7/GFP fusion gene bearing the +2.8- to + 5.3-kb unc-86 segment is expressed in the URX, RIH, and reiterated PQR neurons in an unc-86(n846) animal. The expression in RIH and RIR by fusion genes bearing this segment differs from the expression of the $\mathrm{C}_{34} \mathrm{WXYZ}$ fusion gene that also bears this unc-86 segment. Either orientation of this segment upstream of mec-7/GFP confers expression in these neurons. It is possible that either the location of this segment 3 ' to a proximal element in $\mathrm{C}_{34} \mathrm{WXYZ}$, or the distinct proximal element in this reporter gene, accounts for this difference in expression. 

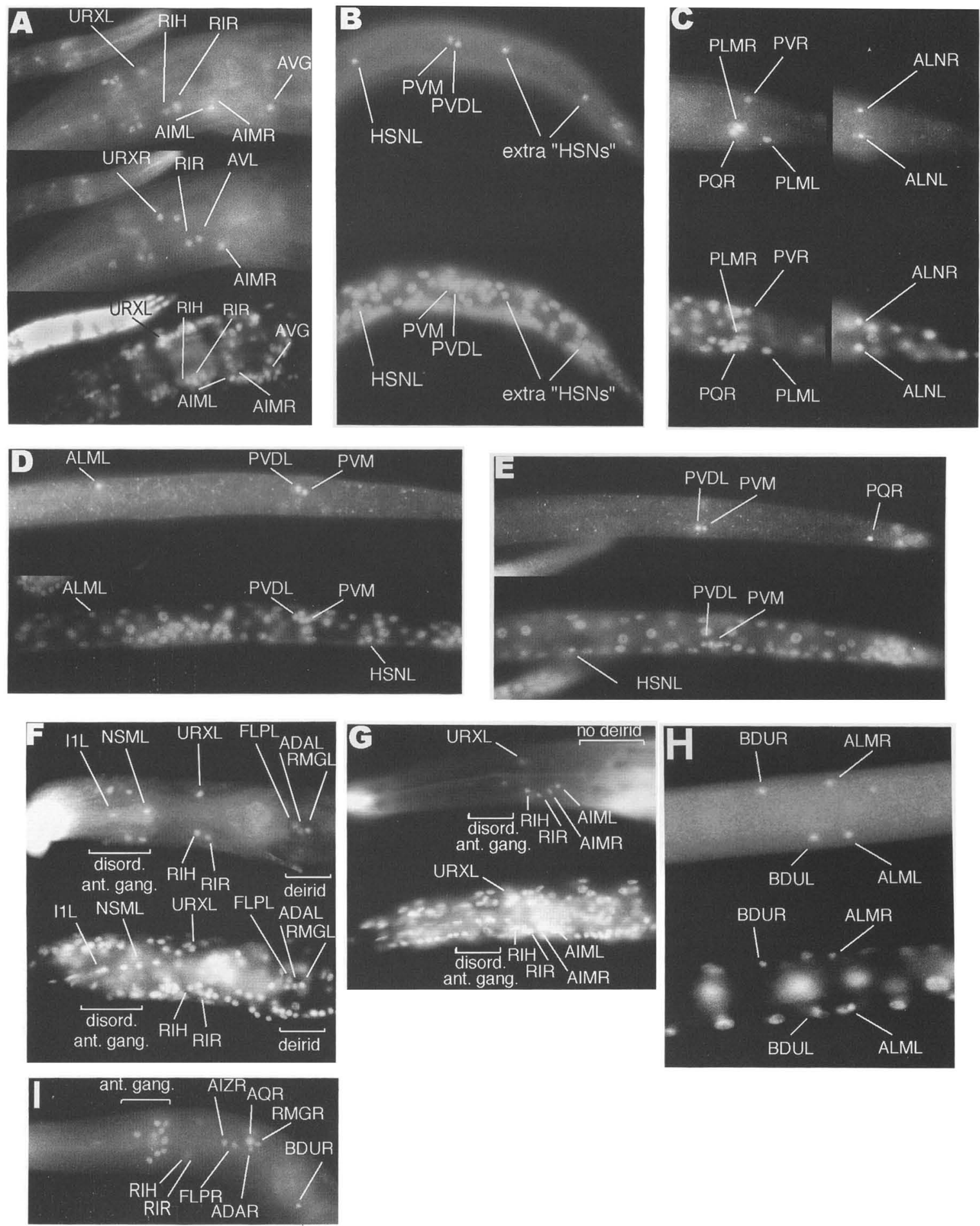

Figure 5. Regulators of unc-86 expression. (Top) Anti-UNC-86 antibody staining in various mutants. (Bottom) DAPI. (A) lin-11(n389). (Top and center) Two focal planes of head region; ectopic unc-86 expression in AVG and the putative AVL. $(B)$ Left lateral view of ham-1(n1810) tail (left focal plane). Two additional unc-86-expressing cells are found between PVM/PVDL (postdeirid) and tail neurons (right). The position of HSNL is indicated. (C) Dorsal view of lin-17/n671) tail. Only six cells express UNC-86, PHC(L/R) and PLN(L/R) expression is missing. (Left) ventral plane; (Right) dorsal plane. (D,E) egl-5/n486) animals do not activate unc-86 expression in the HSN neurons. In $D$, the HSN shows a migration defect as well as a failure to activate unc-86 expression. In $E$, the HSN migrates normally but does not activate unc-86 expression. $(F)$ vab-3(e1062) show disordered anterior ganglion and missing neurons that normally express unc-86. unc-86 expression in the URX, RIH, RIR, and deirid neurons is normal. (G) lin-32/u282). Few neurons in the anterior ganglion express unc-86. Deirid neurons are absent. The six IL2 neurons are either missing or do not express Unc-86. Although additional cells are probably also affected, they could not be identified unambiguously because of a general displacement of neurons in the anterior ganglion. There is normal unc-86 expression in RIH, RIR, and AIM neurons. (H) ALM migration defect in lin-32(u282) animals; dorsal view. (I) For comparison, the normal pattern of unc-86 expression in the head of a wild-type animal, right focal plane. 
Figure 6. unc-86 expression defects in various mutants. The cell-lineage-specific pattern of unc-86 activation in each mutant background is shown using the lineages from Fig. 1 arrayed in anterior to posterior location. All of the mutants tested are non-null loss-of-function (lf) mutants with the exception of mab-5(e1751) (gainof-function: gf) and lin-44(n1792), which is a null mutant. unc-86-expressing cells are shown as in Fig. 3. (O) Number and position of unc-86-expressing cells is identical to wild type. (O) Reduced number of cells express in the particular lineage. (Left and right arrowheads) Neuron mispositioned to the anterior/posterior. (Boxes) Reduced number of neurons expressed in the anterior ganglion; identity of these cells was not determined.

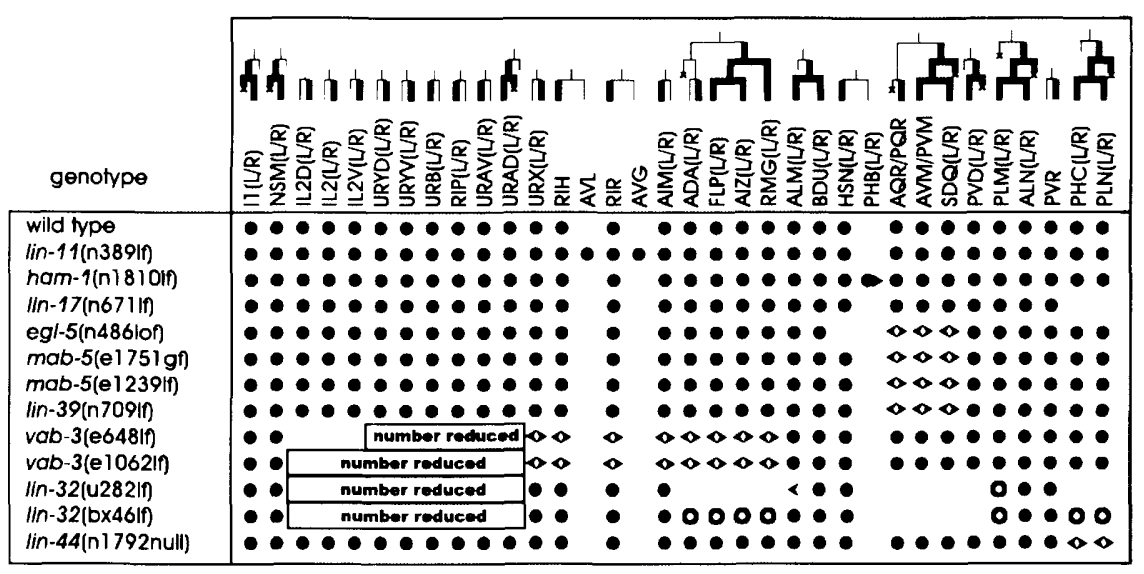

ably by repressing unc-86 expression in AVG. The pattern of 1 in-11 expression has not been reported so it is not yet known if it is expressed in AVG or RIR. Infrequently $\mid<10 \%$ penetrance), one additional cell expresses unc-86 in the right, posterior section of the ventral ganglion (Fig. 5A). We have not identified this cell, but suggest that it might be AVL, which is the only unilateral cell in this position and is the sister of the unc-86-expressing interneuron RIH.

ham-1 controls the asymmetry of unc- 86 expression in the HSN cell lineages

ham-1 controls the asymmetric division of the neuroblast that normally generates the neurons HSN and PHB. Mutations in ham-1 cause a partially penetrant transformation of HSN sister cells PHB into HSNs as well as migration defects in HSN neurons (Desai et al. 1988). To determine whether ham-1 acts upstream or downstream of unc-86, and whether it controls other cell lineage asymmetries mediated by unc- 86 , we observed the pattern of unc-86 expression in animals bearing the strong ham-1(n1810) allele. We detect up to five extra unc-86expressing neurons in the region through which the HSN migrates in ham-1(n1810) animals (142 animals; Figs. 5B and 6). These extra unc-86-expressing neurons occasionally show an increase in UNC-86 protein level at late L4 and adult stages, which is a hallmark of HSN neural fate (Finney and Ruvkun 1990), and suggests that these extra neurons are partially specified HSN neurons. These extra HSN-like unc-86-expressing neurons are likely to result from the symmetric activation of unc-86 in both daughters of the HSN/PHB parent neuroblast, specifying both daughters toward the HSN fate. These data suggest that ham-1 acts upstream of unc-86 and, directly or indirectly, mediates the asymmetric expression of unc-86 in the HSN/PHB neuroblast cell lineage. The fact that we observed up to five HSN-like neurons is consistent with data from G. Garriga that ham-1 not only regulates the asymmetry of the neuroblast parent to HSN and PHB, but earlier divisions of this lineage as well /G. Garriga, pers. comm.). No other perturbations to the asymmetric unc-86 expression pattern were observed in ham-1(n1810), suggesting that ham-1 does not function in the other neuroblast cell lineages that asymmetrically activate unc-86 expression.

lin-17 and lin-44 act upstream of unc-86 expression in the $T$-cell lineage

lin-17 and lin-44 mutations disrupt the $\mathrm{T}$ lineage one or more rounds of division before unc-86 is normally expressed. In lin-17 mutants, a symmetrization of the T.a and T.p division generates two identical daughters that behave as T.a, resulting in four missing tail neurons (Sternberg and Horvitz 1988). Consistent with this observation, only six neurons express unc-86 in the tail of lin-17(n671) animals (6.3 \pm 0.4 cells; 36 animals) rather than 10 neurons in wild-type animals (Figs. $5 \mathrm{C}$ and 6 ). No other unc-86 expression defects were observed in lin17(n671) mutants. Way et al. (1992) have reported that at low penetrance $\operatorname{lin}-17($ n671) mutant animals have additional mec-3-expressing sister cells of PVD, PVM, PLM, and FLP neurons. unc-86 expression is not affected in these lineages. These results suggest that $l i n-17$ functions before unc- 86 expression in the $\mathrm{T}$ lineage but does not act upstream of unc-86 in other cell lineages. Similarly, effects on the regulation of unc-86 expression only in the $\mathrm{T}$ cell lineage were observed in a lin-44 null mutant (Herman and Horvitz 1994) (Fig. 6).

The HOM-C cluster gene egl-5 is necessary for the activation of unc-86 expression in the HSN neuron

The HOM-C gene egl-5, a probable homolog of Drosophila Abdominal- $B(A b d-B)$ (Wang et al. 1993) acts upstream of unc-86 in the specification of the HSN neuron. egl-5 controls the specification of a variety of cells in the posterior body region including the HSN neuron (Desai et al. 1988; Chisholm 1991; Clark et al. 1993). In animals 
bearing the strong egl-5(n486) allele, the presumptive HSNs are generated normally but are defective in migration as well as serotonin synthesis, and the animals are egg-laying defective (Desai et al. 1988). More than 95\% of the egl-5(n486) animals $(n=111$ of 115$)$ do not express unc- 86 in the HSN neuron, indicating that egl-5 acts upstream of unc- 86 and is a potential regulator of unc-86 expression in the HSNs (Figs. 5D, E).

unc-86 expression in cells other than the HSNs was not affected in the egl-5 mutant. However, in some cases the migration of neurons generated from the $\mathrm{Q}$ lineage is disturbed (Fig. 6). Mutations in other HOM-C genes (mab-5, lin-39) do not result in unc-86 expression defects but lead to migration defects of the $\mathrm{Q}$ lineage (Kenyon 1986; Chisholm 1991; Salser and Kenyon 1992; Clark et al. 1993; Wang et al. 1993) (Fig. 6).

vab-3, the C. elegans homolog of Pax-6, is necessary for unc-86 expression in sensory head neurons

$v a b-3$ mutants are defective in several sensory functions including chemosensation and thermotaxis (Lewis and Hodgkin 1977). vab-3 is the C. elegans gene homolog of Pax-6/eyeless, which specifies eye development in vertebrates and invertebrates (Chisholm and Horvitz 1995; Zhang and Emmons 1995). The strong vab-3 alleles $e 648$ and $e 1062$ result in missing unc-86 expression in several neurons in the head. On average, 10 cells $\langle 10.3 \pm 1.6 ; 60$ animals) in $v a b-3(e 1062)$ and $12(12.1 \pm 1.7 ; 59$ animals) in $v a b-3(e 648)$ express unc-86 in the anterior ganglion, compared with $18.0 \pm 0.04$ (150 animals) in wild type (Figs. 5F and 6). The identity of the neurons that do not express unc-86 could not be determined, as the overall anatomy of the anterior ganglion is severely disturbed in these mutants. However, all missing neurons are normally generated in the anterior sensory regions of the head, suggesting that vab-3 acts in a particular anterior spatial domain. vab-3 function in tail development has been described (Chow and Emmons 1994; Zhang and Emmons 1995), but expression of unc-86 in the vab-3 mutant tail was normal. Therefore vab-3 acts upstream of unc-86 expression in the generation or differentiation of neurons of the anterior ganglion.

\section{lin-32 acts upstream of unc- 86 in sensory neural cell lineages}

lin-32 encodes a basic-helix-loop-helix protein similar to Drosophila atonal and is necessary for the specification of neuroblast fate in several lineages (Zhao and Emmons 1995). In animals bearing the strong lin-32(u282) allele (Zhao and Emmons 1995), the Q and V5 neuroblasts are transformed to ectodermal cells (Chalfie and Au 1989; Zhao and Emmons 1995). Consistent with a lin-32 function in the generation of the neuroblasts that express unc-86, no unc-86 expression was observed in these transformed ectoblast cell lineages (data not shown). We observe other defects in the unc-86 expression pattern in the neurons of the anterior ganglion, the deirid, and the tail (Fig. 5G). unc-86 expression in other cells, for example, HSN, BDU, and ALM was not affected by the lin-32(282) mutation, although the latter are frequently displaced (Fig. 5H). Therefore, lin-32 acts upstream of unc-86 in many but not all of the neuroblast cell lineages that express unc-86.

\section{Discussion}

All of the 302 neurons of the C. elegans nervous system are generated from neuroblast cell lineages that are asymmetric (Sulston and Horvitz 1977; Sulston et al. 1983). The POU homeo box gene unc-86 couples cell lineage asymmetry to the generation of neuron and neuroblast identity in 27 of the 118 classes of neurons (Finney and Ruvkun 1990). Asymmetric activation of unc-86 expression may be an initial step in a cascade of transcriptional regulation during neuron and neuroblast differentiation (Way and Chalfie 1989; Hamelin et al. 1992).

\section{Establishment of unc-86 expression is transcriptional and modular}

We show here that the transcriptional regulatory region of unc-86 detects asymmetries in cell lineages to activate unc-86 expression in particular daughter neuroblasts and neurons. Segments from both the $5^{\prime}$ and $3^{\prime}$ unc-86 regulatory region are necessary and sufficient for the activation of asymmetric unc-86 expression (Fig. 3). Furthermore, distinct segments of the unc- 86 regulatory region respond to asymmetric cues only in particular neural lineages (Fig. 3). This analysis suggests that the distinct transcriptional regulators of unc-86 expression in these neural lineages are either asymmetrically segregated or differentially activated (or inactivated) in these asymmetric cell divisions. For example, asymmetrically activated transcription factors in the RIH and RIR cell lineages act through enhancers located between -2.7 to $-0.8 \mathrm{~kb}$ and +2.8 to $+5.3 \mathrm{~kb}$ in the unc- 86 promoter, whereas transcription factors that are activated in the Il, NSM, V5.paa, T.ppp, and Q.p cell lineages act through enhancers located between -5.1 to $-2.8 \mathrm{~kb}$ (Fig. 3). In other cell lineages, our analysis suggests that distinct transcription factors bind to sites located in multiple unc-86 regulatory regions (Fig. 3). Our analysis does not distinguish whether distinct factors act in each of the cell lineages or whether distinct combinations of factors interact on the unc-86 promoter in particular cell lineages. Even if there are regulators common to these cell lineages, the observation that unc- 86 regulatory regions respond to distinct cell lineage-specific cues for the asymmetric activation of the gene shows that there must be key regulators whose activation is specific to the particular cell lineages. This logic is consistent with the analysis of transregulators, which demonstrates regional and cell lineage specificity.

Redundant regulatory regions for maintenance of unc-86 expression

Expression of all unc-86 promoter-reporter genes is tran- 
sient in an unc-86 null mutant and fades soon after the expression is initiated. In contrast, the expression of the same genes is maintained when transformed into wildtype animals that express a functional UNC-86 protein. These results indicate that unc-86 is necessary, either directly or indirectly, for its own maintained expression. Stronger evidence for unc-86 autoregulatory function comes from the distinct patterns of expression that most reporter genes show in wild type compared with unc-86 null mutants, where autoregulation is disabled (Figs. 3 and $4 \mathrm{HI})$. Autoregulatory segments are located both $5^{\prime}$ and 3 ' of the transcriptional start and are redundant, because any one of these segments is sufficient to confer unc-86-regulated fusion gene expression in all neurons that normally express the gene. unc-86 autoregulation functions independently from unc-86 establishment. For example, the fusion gene $\mathrm{C}_{34} \mathrm{WXYZ}$ can initiate unc-86 expression in only 19 neurons but responds to unc-86 autoregulation in all 57 neurons (Fig. 4H,I).

unc-86 encodes a transcription factor and therefore is a likely candidate to directly autoregulate its own expression (Finney et al. 1988; Xue et al. 1992). Analogous autoregulation of the POU gene Pit-1 has been observed (Chen et al. 1990). By sequence analysis, there are seven consensus UNC-86 binding sites in the $10.4-\mathrm{kb}$ unc-86 gene fragment, based on those detected biochemically in mec-3 and brn-3 (Xue et al. 1992; Li et al. 1993) (Fig. 3A). unc- 86 autoregulation could also be achieved by an indirect mechanism (Bienz 1994).

The unc-86 autoregulation we observe is inconsistent with the previous observation of normal UNC-86 expression level in strains carrying strong unc-86 alleles that make immunodetectable protein (Finney and Ruvkun 1990). There are several possible explanations for this discrepancy. For example, if in addition to positive autoregulation, unc-86 also negatively autoregulates its expression, as has been found for Pit-1 (Chen et al. 1990), unc-86 mutants may compensate for a decrease in unc86 activity with an increase in expression of a nonfunctional protein. On the other hand, the particular mutant Unc- 86 proteins tested to date might function normally for autoregulation, but not in the specification of neuron and neuroblast identity. Alternatively, there may be other positive regulatory sites not present on the fusion genes tested that activate unc-86 expression in a nonunc-86-dependent manner. A less likely alternative explanation is that either wild-type or mutant UNC-86 is a stable protein.

The analysis of the unc-86 promoter in both wild-type and unc-86 mutants reveal two aspects to unc-86 asymmetric activation. Initially, the complex unc-86 regulatory region builds up the pattern of unc- 86 expression piece by piece using regulatory pathways that are unique to particular sets of cell lineages. As described below, these sets of cell lineages are spatially clustered suggesting that spatially localized cues activate particular transcription factors that bind to corresponding unc-86 regulatory regions to activate unc-86 expression. Once expression of unc- 86 is initiated, unc- 86 autoregulation can confer continued UNC-86 expression. In this way, the initial activators of asymmetric unc-86 expression may be active only transiently to trigger unc- 86 autoregulation.

Asymmetric regulation of unc- 86 is controlled by genes specific to particular cell lineages

The analysis of unc-86 transcriptional regulatory sequences predicts that distinct transcription factors activate unc-86 expression in particular cell lineages. In agreement with such a model, we found mutations in a set of transcription factor and cell lineage control genes that perturb the asymmetric activation of unc-86 in a subset of the cell lineages that normally express the gene. For example, a lin-11 mutant shows symmetric unc-86 expression in the interneuron AVG as well as in its sister neuron RIR, which normally expresses unc-86. Therefore, lin-11 may function in the neuroblast parent of RIR and AVG to generate asymmetric daughters that express distinct sets of genes, including unc-86 in RIR only. In such a model, lin-11 would function before unc86 in this particular cell lineage, but not necessarily as a direct regulator of unc-86 expression. However, it is also possible that lin-11 may function in AVG to repress unc86 expression. Like the unc-86 downstream gene mec-3, whose gene product interacts with UNC- 86 protein to regulate maintenance of $\mathrm{mec}-3$ gene expression (Xue et al. 1993), lin-11 encodes a LIM homeo domain protein (Freyd et al. 1990). A model that LIN-11 normally antagonizes the UNC-86 autoregulatory activity in the AVG neuron is appealing because of the known association of POU and LIM proteins in maintenance of mec-3 expression (Xue et al. 1993). However, our data do not favor a function for lin-11 in the prevention of unc-86 maintenance because we have not observed transient unc-86 expression in AVG in wild-type or unc-86 mutants ( $R$. Baumeister and G. Ruvkin, unpubl.). We, therefore, favor the model that LIN-11 either functions in the neuroblast parent of AVG and RIR, or in AVG to inhibit the function of an unidentified transcription factor that can activate unc-86 expression in both RIR and AVG. There is precedent for such combinatorial control of neurogenesis by LIM proteins (Tsuchida et al. 1994; Bach et al. 1995).

ham -1 controls asymmetric activation of unc- 86 in the HSN/PHB cell lineage. It is probable that ham-1 represses unc-86 in the PHB neuron, or acts earlier to specify an asymmetric division by the neuroblast parent of HSN and PHB. It is not known whether ham-1 encodes a direct transcriptional regulator analogous to $\operatorname{lin}-11$ or whether it regulates the activity of such a regulator.

ham-1 and lin-11 appear to act as repressors of unc-86 expression in these two cell lineages. On the other hand, the rather large DNA segments used in the unc-86 promoter analysis revealed only examples of positive regulation of unc-86 activation (Fig. 3). Only high copy transgenes revealed other examples of possible unc-86 repression in the particular cell lineages (Fig. 4G). It is possible that negative regulatory elements in the unc-86 regulatory region, for example, that interact with LIN-11 or HAM-1, are closely associated with positive elements, as 
has been observed in the case of Drosophila eve (Stanojevic et al. 1991).

Our analysis of other mutants revealed candidates to mediate the positive regulation of unc-86 in particular cell lineages. For example, a mutant in the HOM-C gene egl-5 generates HSN neurons that do not express unc-86. unc-86 regulation in the other 55 neurons that express the gene is normal in this egl-5 null mutant. Therefore egl-5 acts upstream of unc-86 only in the HSN. egl-5 affects a broader range of HSN functions, consistent with it acting upstream of unc-86 in HSN specification (Desai et al. 1988). The other HOM-C genes, mab-5 and lin-39, do not affect unc-86 expression. Therefore each HOM-C gene is unlikely to provide the spatial cues for unc-86 up-regulation that we noted from the unc-86 promoter analysis. However, given that the expression of the HOM-C genes overlaps in some cells and evidence for combinatorial control of cell fate by this gene cluster, it is possible that analysis of animals mutant for multiple members of the HOM cluster genes might show abnormal activation of unc-86 in the spatial domains specified by these genes.

Mutations in lin-32, a member of the achaete/scute $(a c / s c \mid$ class of neurogenic transcription factor, and $v a b$ 3, a Pax-6 homolog, affect several neural lineages which produce unc-86-expressing sensory neurons but do not affect other unc-86-dependent cell lineages (Fig. 6). All of the lineages affected by these genes produce sensory neurons, consistent with the function of their homologs in other animals (Jarman et al. 1993; Glaser et al. 1994; Halder et al. 1995). However, because only non-null alleles of these genes have been isolated, we cannot yet rule out that the unaffected cell lineages have a lower threshold for lin-32 or vab-3 gene activity. Although the effects of these mutants on unc-86 expression may reflect an earlier function in neuroblast specification, it is also possible that each of these transcription factors directly regulates unc- 86 .

These results suggest that cell lineage-specific regulators bind to distinct enhancers distributed across the unc-86 regulatory region. The enhancer elements that respond to HAM-1 and EGL-5 are likely to be located in the $-5.1-$ to $-2.8-\mathrm{kb}$ and $+2.8-$ to $+5.3-\mathrm{kb}$ segments that establish unc-86 expression in the HSN cell lineage. The unc- 86 regulatory region that responds to LIN-11 (or a gene regulated by LIN-11) to silence unc-86 expression in AVG may correspond to the $-2.6-$ to $-0.8-\mathrm{kb}$ and +2.8 - to $+5.3-\mathrm{kb}$ positive regulatory unc- 86 segments that establish expression in RIR, the sister of AVG (Fig. 3A).

unc-86 integrates spatial and cell lineage control signals

Asymmetric activation of unc- 86 is transcriptional and occurs within $10 \mathrm{~min}$ of the birth of an unc-86-expressing neuroblast or neuron (Finney and Ruvkun 1990). We suspect therefore that particular transcription factors inherited from the mother of that neuroblast or neuron are either asymmetrically segregated to, or activated in, one daughter cell to activate unc- 86 expression. Therefore, any mechanism that establishes this asymmetry acts at the division of the mother cell to regulate the dowry of transcription factors inherited by unc-86-expressing daughter cells. For that reason, we inspected the location of the mothers of unc-86-expressing cells (Fig. 7) generated in embryogenesis.

We found that the mothers of the unc-86-expressing neurons or neuroblasts are clustered at various positions in the embryo. This is most obvious in the anterior half of the embryo, where these mother cells form stripes perpendicular to the antero-posterior axis (Fig. 7). The spatial clusters of neuroblasts correspond quite accurately with the sets of neurons whose unc-86 expression is regulated by particular segments in the unc- 86 promoter. For example, gene segments that activate unc- 86 expression in the neurons of the anterior ganglion are always accompanied by expression of the AIM neurons in the ventral ganglion, which is located further posterior in an Ll animal (Figs. 6 and 7). In the embryo, the mother cells of AIM and those of RIP, URYV, IL2V, and URAV are all located in the same antero-posterior position in the embryo.

We suggest that all the unc-86-expressing cells derived from this spatial cluster use a common regulatory mechanism mediated by particular cis elements in the unc- 86 regulatory region. Consistent with this assumption, mutations in the unc- 86 regulator $v a b-3$ only affect neurons derived from the two anterior-most clusters of cells. In an analogous manner, lin-11 mutants only affect expression of RIH and RIR and their sisters, which are derived from two mother cells that are neighbors in a central position at the ventral side of the embryo.

At least within the two anterior clusters, the polarity of the asymmetry to which the unc-86 promoter responds seem to be already programmed in the mother cells, because the unc-86-expressing daughter cells always arise from the faces of the mother cells which are oriented towards one another. This polarization of mother neuroblasts could be due to an external oriented signal along the line demarked by the oriented neuroblasts or could correspond to an earlier boundary - for example a segment boundary which has been implicated as signaling centers in Drosophila disc development ( Tabata and Kornberg 1994). It is striking that lin-11 is necessary for such a cell lineage polarization, triggered by anchor cell signaling in the developing vulvae (Freyd et al. 1990|. lin-11 could propagate an analogous polarization cue in the cell lineage that generates the AVG/RIR sister neurons.

\section{A common pathway for neurogenesis}

Homologs of unc-86 that are expressed and function in the developing nervous system have been identified in Drosophila (Treacy et al. 1992; Yang et al. 1993), mouse (Xiang et al. 1993), and human (Gerrero et al. 1993). In Drosophila, POU proteins have been shown to function like unc-86 in asymmetric neuroblast specification (Yeo et al. 1995; Bhat et al. 1995). The emerging view (Jan and 


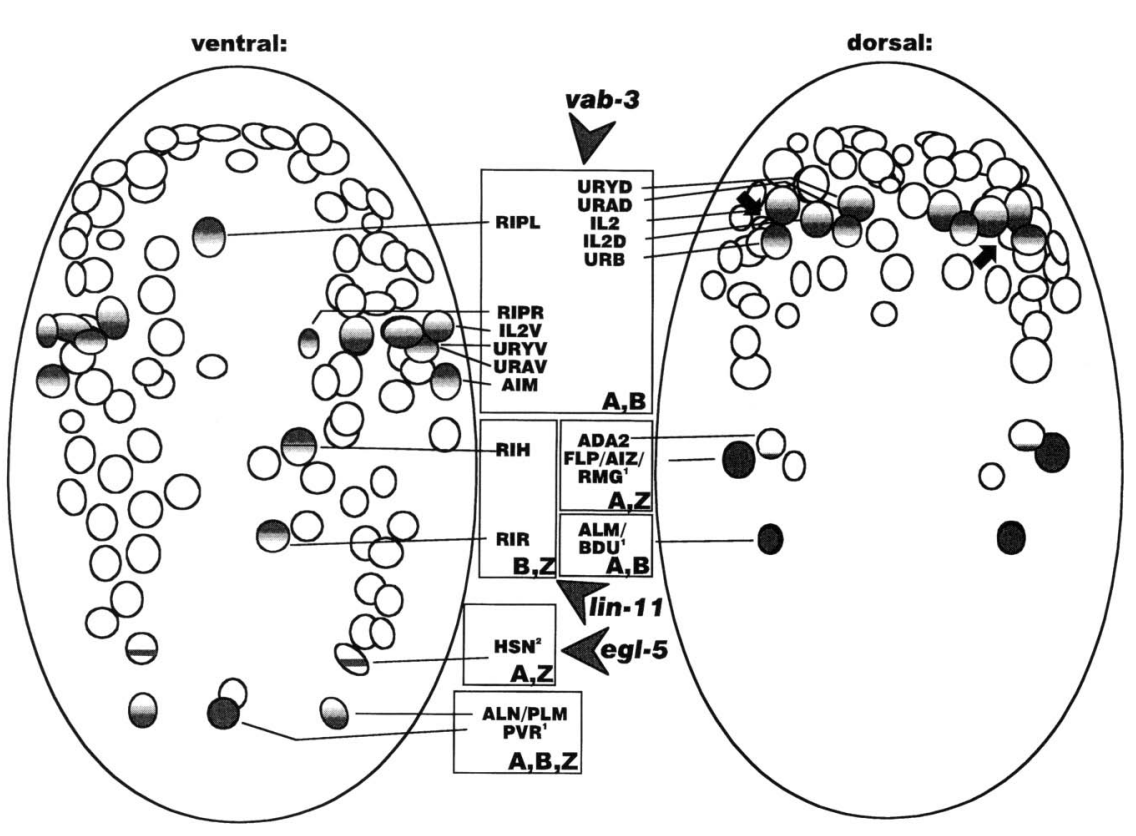

Figure 7. Location of mother cells that activate unc-86 expression asymmetrically in one of two daughters. Neuroblasts only on dorsal and ventral views of a 260 min embryo are shown. Cells whose daughters will express unc-86 are shaded, with the shaded half indicating the position of the daughter after cell division. Only one of two bilaterally symmetric cells is labeled. The clusters of neuroblasts that express fusion genes bearing particular unc-86 gene segments are boxed, with the name of the segments indicated. Genes that affect unc-86 expression in these cells are also indicated. Not shown are the neuroblasts that generate the URX, Il, and NSM neurons and the neuroblasts that divide postembryonically. (Solid arrows) Position of $\mathrm{ADL}(\mathrm{L} / \mathrm{R})$ neurons, where unc-86 expression is regulated negatively (see text). (Footnoted cells) ' Shaded cells already express unc-86 at this stage. They are neurons (PVR) or neuroblasts that will divide again to generate BDU/ ALM and FLP/AIZ/RMG. ${ }^{2}$ The granddaughters of these neuroblasts will express unc-86. The cell positions and names taken from Sulston et al. (1983).
Jan 1994) is that both cellular interactions mediated by a Notch-signaling system coupled to $a c / s c$ transcription factors, and segregated factors, such as Numb and Prospero (Hirata et al. 1995; Knoblich et al. 1995; Spana and Doe 1995) have important roles in the generation of this neural cell lineage asymmetry in Drosophila (Posakony 1994). Homologs of these genes (Bürglin 1994) may mediate aspects of asymmetric unc-86 expression in C. elegans, and of analogous POU proteins across phylogeny.

Homologs of the genes that regulate unc-86 expression in particular subdomains of its expression pattern, the $a c / s c$ gene lin-32, the LIM gene lin-11, the Pax gene vab3 , and the HOM-C gene egl-5 also regulate neurogenesis in vertebrates and other invertebrates (Guillemot et al. 1993; Ferreiro et al. 1994; Glaser et al. 1994). Consistent with a model that in other species, homologs of these genes regulate the expression of unc- 86 homologs, expression of murine Pax-6 in the developing eye (Walther and Gruss 1991) precedes expression of the unc-86 homolog, brn-3 (Xiang et al. 1993). In addition, genes that are regulated by unc-86, for example, the mec-1, mec-4, and mec-7 genes in mechanosensory neurons, are also conserved across phylogeny (Hamelin et al. 1992; Canessa et al. 1993; Huang et al. 1995). Mutations in the human POU gene Brn- 4 cause defects in auditory neurogenesis (de Kok et al. 1995) that may be mechanistically related to the defects in mechanosensory neuron development in unc-86 mutants (Chalfie and Au 1989). Therefore unc- 86 and its homologs may have a universal role in coupling neuroblast cell lineage control signals to the expression of neuron-type specific genes during neurogenesis.

\section{Materials and methods}

Construction of unc-86-GFP and lacZ fusion genes

$\mathrm{ABC}_{1234} \mathrm{WXY}$ was constructed by cloning a SpeI fragment containing the unc- 86 promoter and part of the transcribed region into vector pPD22.04 (Fire et al. 1990). Construct $\mathrm{C}_{34}$ WXYZ deletes sequences upstream of the $E c o R I$ site at -422 and adds a Spel-EcoRI fragment from the unc-86 3' region to the reporter gene downstream of its polyadenylation site. GFP reporter plasmids were constructed by replacing the KpnI-SphI fragment containing the $l a c Z$ gene with the respective GFP reading frame from plasmid TU61 (Chalfie et al. 1994). $\mathrm{ABC}_{1234}$ is a BamHI$X m a I$ deletion of $\mathrm{ABC}_{1234} \mathrm{WXY}$, with fragments $\mathrm{C}_{1234}$ reinserted as a PCR fragment from primers RB15 (5'-CAGGAACTTCAAGGTTGAGGATCC) and RB14 (5'-GGTCATTTTTGAAGCGGTTGTCG). $\mathrm{ABC}_{1234}, 1$ and $\mathrm{ABC}_{1234}, 2$ are $\mathrm{ABC}_{1234}$ with the DsaI-XmaI fragment replaced by a synthetic fragment CACGGACATGACCCC and CACGGAAAAATGACCCC, respectively. $\mathrm{AC}_{1234}$ is a BamHI-BglII deletion construct, and $\mathrm{BC}_{1234}$ is a SaII-BamHI deletion derivative of $\mathrm{ABC}_{1234} \cdot \mathrm{C}_{1234}$ is a HindIII deletion construct derived from $\mathrm{ABC}_{1234}$.

The mec-7/GFP and pes-10/lacZ fusion genes were constructed by ligation of a $2.3-\mathrm{kb}$ SpeI-HindIII fragment $\left(\mathrm{A}^{\prime}\right.$ : $\mathrm{co}^{-}$ ordinates -5.1 - to $-2.8-\mathrm{kb}$ ) or a $1.9-\mathrm{kb}$ HindIII fragment $\left(\mathrm{B}^{\prime}\right.$ : coordinates -2.7 to $-0.8 \mathrm{~kb}$ ) from the unc- 86 upstream region, or a 2.5-kb Spel EcoRI fragment (Z: coordinates +2.6 to +5.2 $\mathrm{kb})$ from the unc- $863^{\prime}$ end into the polylinker of pPD95.25 or a GFP derivative of pPD52.102 (J. Kaplan, pers. comm.). These reporter genes bear proximal elements of the pes-10 and mec-7 genes, respectively, and the unc-86 segments were inserted in either orientation upstream of these proximal elements.

\section{Determination of the unc-86 transcriptional start}

The correct unc-86 mRNA start site was determined by PCR 
amplification of first-strand reverse-transcribed total RNA and RNA-linker ligation (Innis et al. 1990). The longest ligation product starts with the (G)GGACATGC at nucleotide 5095 (numbers according to Fig. 3). Consistent with this result, only primers mapping $3^{\prime}$ to the identified start site allowed amplification of spliced products.

\section{Expression analysis of reporter genes}

Fusion gene DNA was co-injected with visible genetic marker plasmids as described (Mello et al. 1991). Most transformations used $10 \mathrm{ng} / \mu \mathrm{l}$ reporter gene (low-copy number). In transformations that surveyed negative regulatory elements, reporter genes were injected at $200 \mathrm{ng} / \mu \mathrm{l}$ (high-copy number). In general fusion gene expression in a particular neuron or neuroblast was viewed in numerous animals and multiple lines. To ensure a better reproduction of the weak expression levels, some photographs were taken from transgenic animals injected at $200 \mathrm{ng} / \mu \mathrm{l}$.

$l a c Z$ and unc-86 expression was detected as indirect immunofluorescence using anti- $\beta$-galactosidase (Promega) and antiUNC-86 specific antibodies as described (Finney and Ruvkun 1990 . Cells stained with anti-UNC- 86 or anti- $\beta$-galactosidase antibodies were identified based on the position of their nuclei with respect to DAPI staining. GFP expressing cells were identified based on their position and the morphology of cell body and axon. Because of their rapid migration in $\mathrm{L} 1$ and the lack of a fixed final position, the four nuclei of the $\mathrm{T}$ lineage, $\mathrm{PHC}(\mathrm{L} / \mathrm{R})$ and $\operatorname{PLN}(\mathrm{L} / \mathrm{R})$, could not be identified unambiguously in fixed animals. However, the correct number of tail cells expressing the $l a c Z$ gene and the occasional detection of staining in the dying T.pppp cell indicated expression in the $\mathrm{T}$ lineage. One discrepancy from the reported unc-86 expression pattern (Finney and Ruvkun 1990) was detected. The two pharyngeal neurons Il(L/R), but not I2(L/R), as reported, stain with unc-86 antibodies (confirmed by S. Shaham, pers. comm.). Figure 1 contains the corrected expression pattern.

\section{Acknowledgments}

We thank Mike Finney for the determination of the start point of unc-86 transcription, assistance in the design of these experiments, and detailed critiques on the manuscript. We thank Mark Nameroff for construction and analysis of some fusion genes, Marty Chalfie for GFP plasmids, Andy Fire for advice and gene fusion reagents, the C. elegans Genetics Center for strains, Josh Kaplan for assistance in cell identification, and the mec-7/ GFP fusion gene, Gian Garriga, Connie Zhao, and Scott Emmons for unpublished information, Ralf Schnabel for the use of his microscope and Garth Patterson and the other members of the Ruvkun and Kaplan labs for support and comments on the manuscript. R.B. is the recipient of grant Bal364/1-1,2 from the Deutsche Forschungsgemeinschaft. G.R. is supported by an American Cancer Society Faculty Research Award. This work was supported by a McKnight Foundation Development Award.

The publication costs of this article were defrayed in part by payment of page charges. This article must therefore be hereby marked "advertisement" in accordance with 18 USC section 1734 solely to indicate this fact.

\section{References}

Bach, I., S.J. Rhodes, R.V. Pearse, T. Heinzel, B. Gloss, K.M. Scully, P.E. Sawenchenko, and M.G. Rosenfeld. 1995. P-Lim, a LIM homeodomain factor, is expressed during pituitary organ and cell commitment and synergizes with Pit-1. Proc. Natl. Acad. Sci. 92: 2720-2724.

Bhat, K.M., S.J. Poole, and P. Schedl. 1995. The miti-mere and pdml genes collaborate during specification of the RP2/sib lineage in Drosophila neurogenesis. Mol. Cell. Biol. 15: $4052-4063$.

Bienz, M. 1994. Homeotic genes and positional signaling in the Drosophila viscera. Trends Genet. 10: 22-26.

Bürglin, T. 1994. A C. elegans prospero homologue defines a novel domain. Trends Biochem. Sci. 19: 70-71.

Canessa, C.M., J.D. Horisberger, and B.C. Rossier. 1993. Epithelial sodium channel related to proteins involved in neurodegeneration. Nature 361: 467-470.

Chalfie, M. and M. Au. 1989. Genetic control of differentiation of the Caenorhabditis elegans touch receptor neurons. Science 243: 1027-1033.

Chalfie, M., H.R. Horvitz, and J.E. Sulston. 1981. Mutations that lead to reiterations in the cell lineages of $C$. elegans. Cell 24: 59-69.

Chalfie, M., Y. Tu, G. Euskirchen, W.W. Ward, and D.C. Prasher. 1994. Green fluorescent protein as a marker for gene expression. Science 263: 802-805.

Chen, R., H.A. Ingraham, M.N. Treacy, V.R. Albert, L. Wilson, and M.G. Rosenfeld. 1990. Autoregulation of pit-1 gene expression mediated by two cis-active promoter elements. $\mathrm{Na}$ ture 346: 583-586.

Chisholm, A. 1991. Control of cell fate in the tail region of $C$. elegans by the gene egl-5. Development 111: 921-932.

Chisholm, A.D. and H.R. Horvitz. 1995. Patterning of the Caenorhabditis elegans head region by the Pax-6 family member vab-3. Nature 377: 52-55.

Chow, K.L. and S.W. Emmons. 1994. HOM-C/Hox genes and four interacting loci determine the morphogenetic properties of single cells in the nematode male tail. Development 120: 2579-2593.

Clark, S.G., A.D. Chisholm, and H.R. Horvitz. 1993. Control of cell fates in the central body region of $C$. elegans by the homeobox gene lin-39. Cell 74: 43-55.

deKok, Y.J., S.M. van der Maarel, M. Bitner-Glindzicz, I. Huber, A.P. Monaco, S. Malcom, M.E. Pembrey, H.H. Ropers, and F.P. Cremers. 1995. Association between X-linked mixed deafness and mutations in the POU domain gene POU3F4. Science 267: 685-688.

Desai, C., G. Garriga, S.L. McIntire, and H.R. Horvitz. 1988. A genetic pathway for the development of the Caenorhabditis elegans HSN motor neurons. Nature 336: 638-646.

Doe, C.Q., D. Smouse, and C.S. Goodman. 1988. Control of neuronal fate by the Drosophila segmentation gene evenskipped. Nature 333: 376-378.

Ferreiro, B., C. Kintner, K. Zimmerman, D. Anderson, and W.A. Harris. 1994. XASH genes promote neurogenesis in Xenopus embryos. Development 120: 3649-3655.

Finney, M. and G. Ruvkun. 1990. The unc-86 gene product couples cell lineage and cell identity in C. elegans. Cell 63: 895905.

Finney, M., G. Ruvkun, and H.R. Horvitz. 1988. The C. elegans cell lineage and differentiation gene unc-86 encodes a protein with a homeodomain and extended similarity to transcription factors. Cell 55: 757-769.

Fire, A., S. White Harrison, and D. Dixon. 1990. A modular set of $l a c Z$ fusion vectors for studying gene expression in Caenorhabditis elegans. Gene. 93: 189-198.

Freyd, G., S.K. Kim, and H.R. Horvitz. 1990. Novel cysteine-rich motif and homeodomain in the product of the Caenorhabditis elegans cell lineage gene lin-11. Nature 344: 876-879.

Gerrero, M.R., R.J. McEvilly, E. Turner, C.R. Lin, S. O'Connell, 
K.J. Jenne, M.V. Hobbs, and M.G. Rosenfeld. 1993. Brn-3.0: A POU-domain protein expressed in the sensory, immune and endocrine systems that functions on elements distinct from known octamer motifs. Proc. Natl. Acad. Sci. 90: 1084110845.

Glaser, T., L. Jepeal, J.G. Edwards, S.R. Young, J. Favor, and R.L. Maas. 1994. PAX6 gene dosage effect in a family with congenital cataracts, aniridia, anophthalmia and central nervous system defects. Nature Genet. 7: 463-471.

Guillemot, F., L.C. Lo, J.E. Johnson, A. Auerbach, D.J. Anderson, and A.L. Joyner 1993. Mammalian achaete-scute homolog 1 is required for the early development of olfactory and autonomic neurons. Cell 75: 463-476.

Halder, G., P. Callaerts, and W.J. Gehring. 1995. Induction of ectopic eyes by targeted expression of the eyeless gene in Drosophila. Science 267: 1788-1792.

Hamelin, M., I.M. Scott, J.C. Way, and J.G. Culotti. 1992. The mec-7 $\beta$-tubulin gene of Caenorhabditis elegans is expressed primarily in the touch receptor neurons. EMBO J. 11:28852893.

Herman, M.A. and H.R. Horvitz. 1994. The Caenorhabditis elegans gene lin-44 controls the polarity of asymmetric cell divisions. Development 120: 1035-1047.

Hirata, J., H. Nakagoshi, Y. Nabeshima, and F. Matsuzaki. 1995. Asymmetric segregation of the homeodomain protein Prospero during Drosophila development. Nature 377: 627-630.

Huang, M., G. Gu, E.L. Ferguson, and M. Chalfie 1995. A stomatin-like protein necessary for mechanosensation in C. elegans. Nature 378: 292-295.

Innis, M.A., D.H. Gelfand, and J.J. Sninsky. 1990. PCR Protocols: A guide to methods and applications. Academic Press, Inc., San Diego CA.

Jan, Y.N. and L.Y. Jan. 1994. Genetic control of cell fate specification in Drosophila peripheral nervous system. Annu. Rev. Genet. 28: 373-393.

Jarman, A.P., Y. Grau, L.Y. Jan, and Y.N. Jan. 1993. atonal is a proneural gene that directs chordotonal organ formation in the Drosophila peripheral nervous system. Cell 73: 13071321.

Jin, Y., R. Hoskins, and H.R. Horvitz. 1994. Control of type D GABAergic neuron differentiation by $C$. elegans UNC-30 homeodomain protein. Nature 372: 780-783.

Johnson, W.A. and J. Hirsh. 1990. Binding of a Drosophila POUdomain protein to a sequence element regulating gene expression in specific dopaminergic neurons. Nature 343: 467470.

Kenyon, C. 1986. A gene involved in the development of the posterior body region of C. elegans. Cell 46: 477-487.

Knoblich, J.A., L.Y. Jan, and Y.N. Jan. 1995. Asymmetric segregation of Numb and Prospero during cell division. Nature 377: 624-627.

Lewis, J.A. and J.A. Hodgkin. 1977. Specific neuroanatomical changes in chemosensory mutants of the nematode Caenorhabditis elegans. J. Comp. Neurol. 172: 489-510.

Li, P., X. He, M.R. Gerrero, M. Mok, A. Aggarwal, and M.G. Rosenfeld. 1993. Spacing and orientation of bipartite DNA. binding motifs as potential functional determinants for POU domain factors. Genes \& Dev. 7: 2483-2496.

McIntire, S.L., E. Jorgensen, and H.R. Horvitz. 1993. Genes required for GABA function in Caenorhabditis elegans. $\mathrm{Na}$ ture 364: 334-337

Mello, C.C., J.M. Kramer, D. Stinchcomb, and V. Ambros. 1991. Efficient gene transfer in C. elegans: Extrachromosomal maintenance and integration of transforming sequences. EMBO I. 10: 3959-3970.

Miller, D.M., M.M. Shen, C.E. Shamu, T.R. Burglin, G. Ruvkun,
M.L. Dubois, M. Ghee, and L. Wilson. 1992. C. elegans unc-4 gene encodes a homeodomain protein that determines the pattern of synaptic input to specific motor neurons. Nature 355: 841-845.

Posakony, J.W. 1994. Nature versus nurture: Asymmetric cell divisions in Drosophila bristle development. Cell 76: 415418.

Ruvkun, G. and M. Finney. 1991. Regulation of transcription and cell identity by POU domain proteins. Cell 64: 475-478.

Salser, S.J. and C. Kenyon. 1992. Activation of a C. elegans Antennapedia homologue in migrating cells controls their direction of migration. Nature 355: 255-258.

Salser, S.J., C.M. Loer, and C. Kenyon. 1993. Multiple HOM-C gene interactions specify cell fates in the nematode central nervous system. Genes \& Dev. 7: 1714-1724.

Skeath, J.B., G. Panganiban, J. Selegue, and S.B. Carroll. 1992. Gene regulation in two dimensions: The proneural achaete and scute genes are controlled by combinations of axis-patterning genes through a common intergenic control region. Genes \& Dev. 6: 2606-2629.

Spana, E.P. and C.Q. Doe. 1995. The Prospero transcription factor is asymmetrically localized to the cell cortex during neuroblast mitosis in Drosophila. Development 121:31873195.

Stanojevic, D., S. Small, and M. Levine. 1991. Regulation of a segmentation stripe by overlapping activators and repressors in the Drosophila embryo. Science 254: 1385-1387.

Sternberg, P.W. and H.R. Horvitz. 1988. lin-17 mutations of Caenorhabditis elegans disrupt certain asymmetric cell divisions. Dev. Biol. 130: 67-73.

Sulston, J.E. and H.R. Horvitz. 1977. Postembryonic cell lineages of the nematode Caenorhabditis elegans. Dev. Biol. 56: 110-156.

Sulston, J.E., E. Schierenberg, J.G. White, and J.N. Thomson. 1983. The embryonic cell lineage of the nematode Caenorhabditis elegans. Dev. Biol. 100: 64-119.

Tabata, T. and T.B. Kornberg. 1994. Hedgehog is a signaling protein with a key role in patterning Drosophila imaginal discs. Cell 76: 89-102.

Treacy, M.N., L.I. Neilson, E.E. Turner, X. He, and M.G. Rosenfeld. 1992. Twin of I-POU: A two amino acid difference in the I-POU homeodomain distinguishes an activator from an inhibitor of transcription. Cell 68: 491-505.

Tsuchida, T., M. Ensini, S.B. Morton, M. Baldassare, T. Edlund, T.M. Jessell, and S.L. Pfaff. 1994. Topographic organization of embryonic motor neurons defined by expression of LIM homeobox genes. Cell 79: 957-970.

Walther, C. and P. Gruss. 1991. Pax-6, a murine paired box gene, is expressed in the developing CNS. Development 113: 1435-1449.

Wang, B.B., M.M. Müller-Immerglück, J. Austin, N.T. Robinson, A. Chisholm, and C. Kenyon. 1993. A homeotic gene cluster patterns the anteroposterior body axis of C. elegans. Cell 74: 29-42.

Way, J.C. and M. Chalfie. 1989. The mec-3 gene of Caenorhabditis elegans requires its own product for maintained expression and is expressed in three neuronal cell types. Genes \& Dev. 3: 1823-1833.

Way, J.C., J.Q. Run, and A.Y. Wang. 1992. Regulation of anterior cell-specific mec-3 expression during asymmetric cell division in C. elegans. Dev. Dynam. 194: 289-302.

Xiang, M., L. Zhou, Y.W. Peng, R.L. Eddy, T.B. Shows, and J. Nathans. 1993. Brn-3b: A POU domain gene expressed in a subset of retinal ganglion cells. Neuron 11: 689-701.

Xue, D., M. Finney, G. Ruvkun, and M. Chalfie. 1992. Regulation of the mec-3 gene by the $C$. elegans homeoproteins 
Baumeister et al.

UNC-86 and MEC-3. EMBO J. 11: 4969-4979.

Xue, D., Y. Tu, and M. Chalfie. 1993. Cooperative interactions between the Caenorhabditis elegans homeoproteins UNC86 and MEC-3. Science 261: 1324-1328.

Yang, X., S. Yeo, T. Dick, and W. Chia. 1993. The role of a Drosophila POU homeo domain gene in the specification of neural precursor cell identity in the developing embryonic central nervous system. Genes \& Dev. 7: 504-516.

Yeo, S.L., A. Lloyd, K. Kozak, A. Dinh, T. Dick, X. Yang, S. Sakonju, and W. Chia. 1995. On the functional overlap between two Drosophila POU homeo domain genes and the cell fate specification of a CNS neural precursor. Genes \& Dev. 9: 1223-1236.

Zhang, Y. and S.W. Emmons. 1995. Specification of sense-organ identity by a Caenorhabditis elegans Pax-6 homologue. $\mathrm{Na}$ ture 377: 55-59.

Zhao, C. and S.W. Emmons. 1995. A transcription factor controlling development of peripheral sense organs in C. elegans. Nature 373: 74-78. 


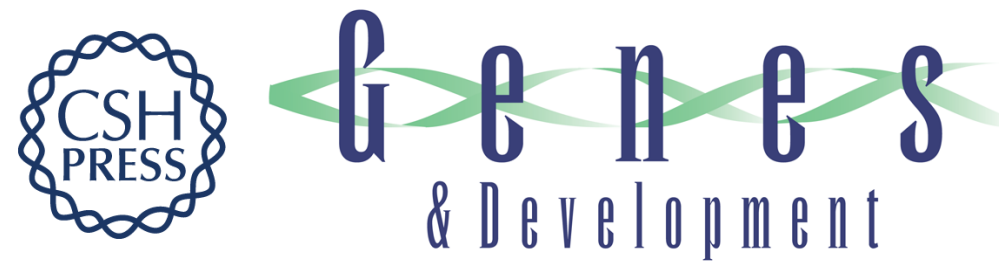

\section{Lineage-specific regulators couple cell lineage asymmetry to the transcription of the Caenorhabditis elegans POU gene unc-86 during neurogenesis.}

R Baumeister, Y Liu and G Ruvkun

Genes Dev. 1996, 10:

Access the most recent version at doi:10.1101/gad.10.11.1395

References This article cites 64 articles, 21 of which can be accessed free at:

http://genesdev.cshlp.org/content/10/11/1395.full.html\#ref-list-1

License

Email Alerting Service

Receive free email alerts when new articles cite this article - sign up in the box at the top right corner of the article or click here.

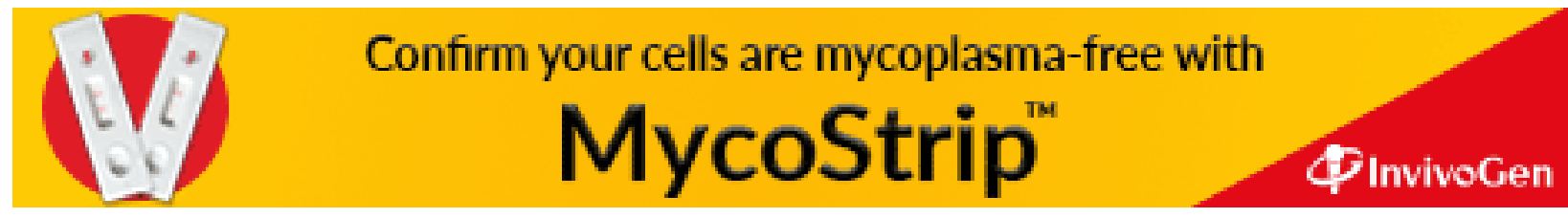

